San Jose State University

SJSU ScholarWorks

Doctoral Projects

Master's Theses and Graduate Research

Spring 4-2016

\title{
A Phenomenological Assessment of Factors that Affect Medication Adherence Among Hypertensive Nigerian Immigrants
}

\author{
Beulah Iroegbu \\ California State University, Northern California Consortium Doctor of Nursing Practice
}

Follow this and additional works at: https://scholarworks.sjsu.edu/etd_doctoral

Part of the Family Practice Nursing Commons, Occupational and Environmental Health Nursing Commons, and the Public Health and Community Nursing Commons

\section{Recommended Citation}

Iroegbu, Beulah, "A Phenomenological Assessment of Factors that Affect Medication Adherence Among Hypertensive Nigerian Immigrants" (2016). Doctoral Projects. 53.

DOI: https://doi.org/10.31979/etd.jcsz-ufj7

https://scholarworks.sjsu.edu/etd_doctoral/53

This Doctoral Project is brought to you for free and open access by the Master's Theses and Graduate Research at SJSU ScholarWorks. It has been accepted for inclusion in Doctoral Projects by an authorized administrator of SJSU ScholarWorks. For more information, please contact scholarworks@sjsu.edu. 


\section{APPROVED \\ For the California State University, Northern Consortium Doctor of Nursing Practice:}

We, the undersigned. certily that the project of the following student meets the required standards of scholarship, tommat, and style of the university and the student's graduate degree program for the awarding of the master's degree.

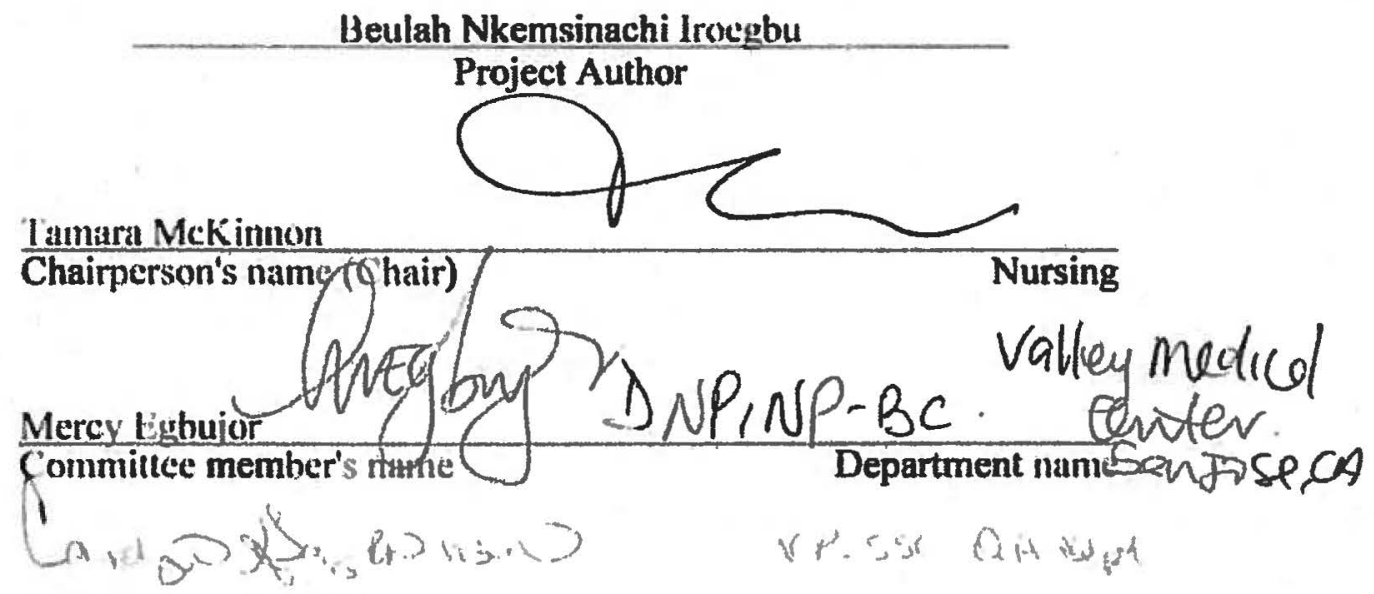

Carolyn Cälndy,RN MSN Kaiser Pernamente South Sacramento Qualits Depl. Committee member's name 
AUTHORIZATION FOR REPRODUCTION OF DOCTORAL PROJECT

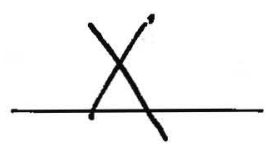

I grant permission for the reproduction of this project in part or in its entirety without further authorization from me, on the condition that the person or agency requesting reproduction absorbs the cost and provides proper acknowledgment of authorship.

Permission to reproduce this project in part or in its entirety must be obtained from me.

Signature of project author:

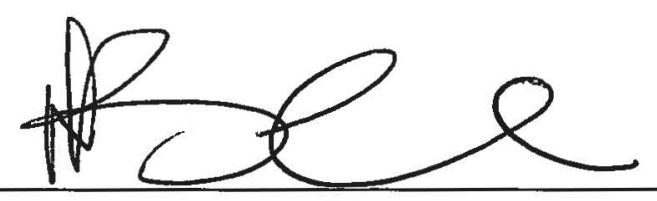


Running Head: HYPERTENSION MEDICATION ADHERENCE

A Phenomenological Assessment of Factors that Affect Medication Adherence Among Hypertensive Nigerian Immigrants

Beulah Iroegbu

California State University, Northern California Consortium

Doctor of Nursing Practice

School of Nursing

April 2016 


\begin{abstract}
This qualitative study explored the perceptions that affect medication adherence among hypertensive Nigerian immigrants in Sacramento, California. The research questions focused on factors that influenced the adherence to hypertension medication; specifically, how the sample population explained the cause of hypertension and whether culture and religion impacted the cause and management of hypertension. A phenomenological approach was used to understand perceptions about medication adherence. A predefined group of 9 acted as a purposive sample and was given semi-structured individual interviews. Qualitative data analysis of the interviews was performed using Colaizzi's 7-step method. Results from the study found the cause of hypertension was believed to be genetics, stress, and diet. Education was strongly cited as the reason for dispelling cultural beliefs toward hypertension cause and management of the illness. Reasons for nonadherence were linked mostly to forgetfulness and side effects of hypertensive medication on sexual performance in males. Participants reported that prayer did not affect medication adherence, but was used for psychological healing. Living in the United States was viewed as a both a positive and negative influence on the management of hypertension.
\end{abstract}

Keywords: hypertension, Nigerian immigrants, adherence, medication. 


\title{
A PHENOMENOLOGICAL ASSESSMENT OF FACTORS THAT AFFECT MEDICATION ADHERENCE AMONG NIGERIAN IMMIGRANTS
}

By

Beulah N. Iroegbu

\begin{abstract}
A Dissertation Submitted to
The California State University, Northern California Consortium.

In Partial Fulfillment of the Requirements for the Degree,

Doctor in Nursing Practice
\end{abstract}

2016

Approved by

Dr. Tamara McKinnon, Project Chair. 


\section{Acknowledgements}

I thank God for this opportunity and express my deepest appreciation to my project chair, Dr. Tamara McKinnon, for guiding and supporting me through this project. Your insightful contributions and direction throughout this process was phenomenal. With a grateful heart, I thank my program advisor, Dr. Christine Ortiz, my mentor, Dr. Christine Lima, my committee members, Dr. Mercy Egbujor, and Carolyn Gandy for your encouragement. I am grateful to Alice Krueger for editing my work and helping me "shine". I thank all my professors for this incredible journey of learning. My cohort and fellow DNP students, I thank you for your inspiration and helping me to overcome difficult times.

I am thankful to Dr. Ernest Uwazie, Mr. Arinze Chukwuneta, and the Nigerian community in Sacramento for your kind support. To all who participated in my study, I offer my deepest appreciation. Words are not enough to express my gratitude to my husband Emenike Iroegbu, my children, Chuks, Ikenna and Uchenna for your unconditional love, support and encouragement throughout this journey. Thank you to my father Ndudim Abraham for your incessant prayers. To the Ndudim, Ukattah, and Okoh families, thank you for all the food you provided and your ever present thoughtfulness. My extended family and friends have been phenomenal throughout my doctoral program, and I thank each and every one of you for your understanding during this time period. 


\section{Table of Contents}

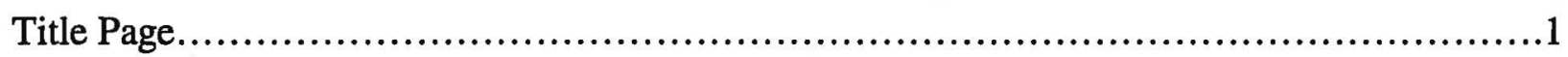

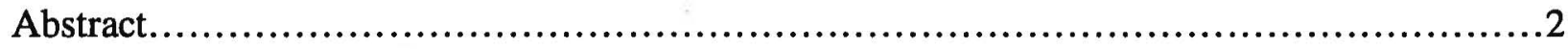

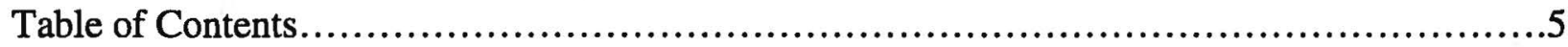

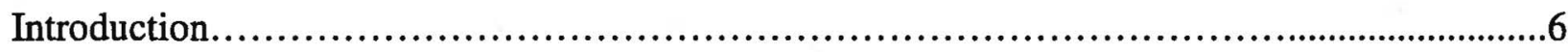

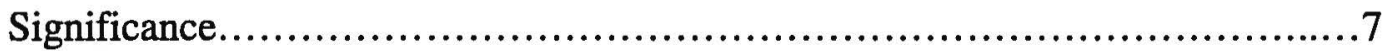

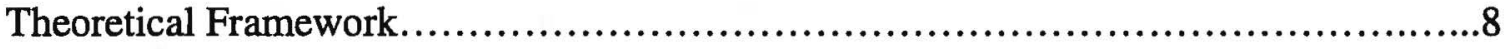

Application of Neuman's Model...........................................9

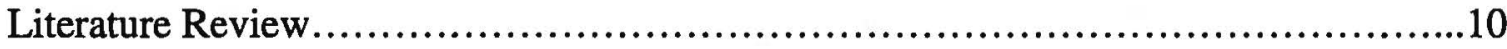

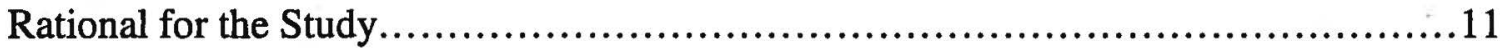

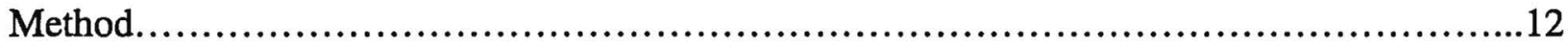

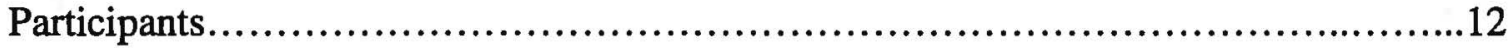

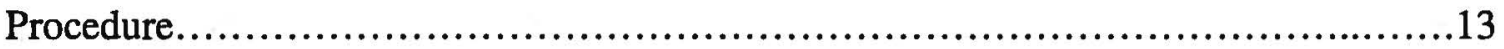

Ethical Consideration...........................................................

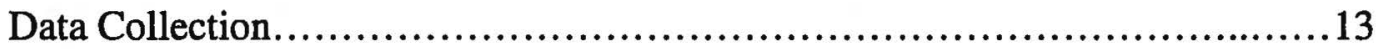

Data Analysis........................................................... 14

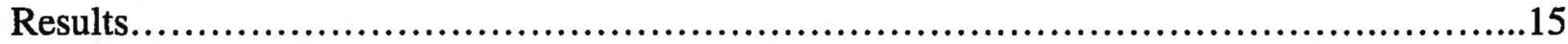

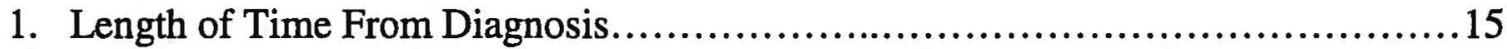

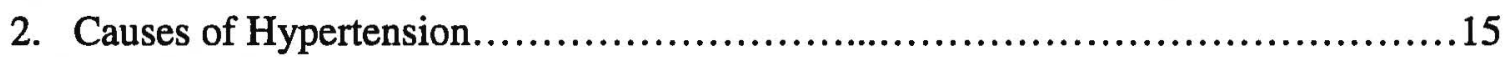

3. Warning Signs of Elevated Blood Pressure .....................................16

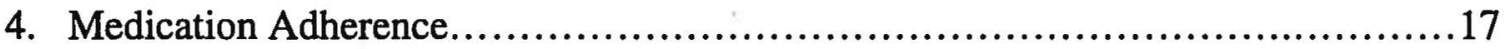

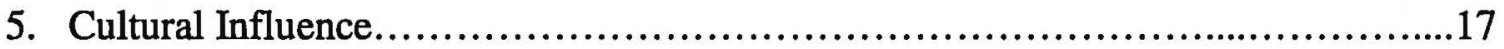

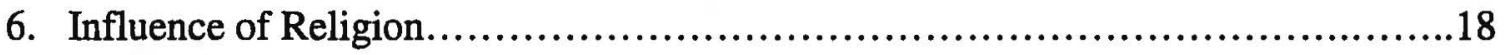

7. Effects of Medication on Sexual Life.............................................18

8. Fears About Hypertension......................................................19

9. Being in the United States.................................................... 19

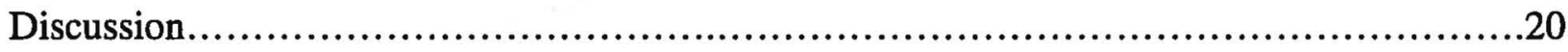

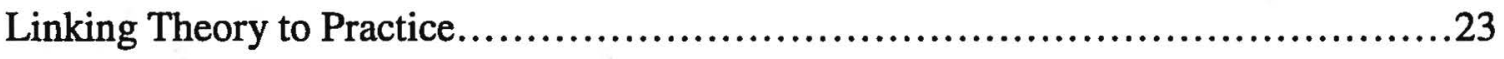

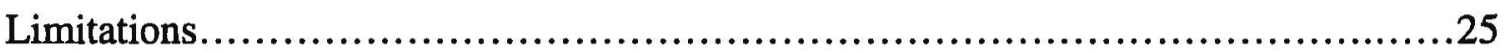

Implications for Nursing Practice and Guidelines..................................25

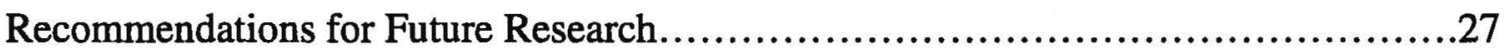

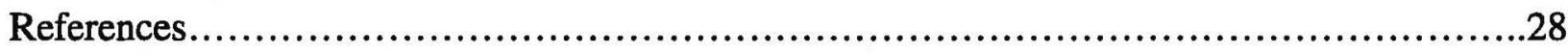

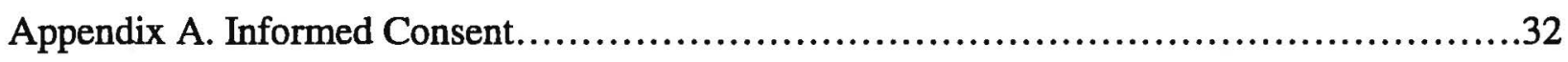

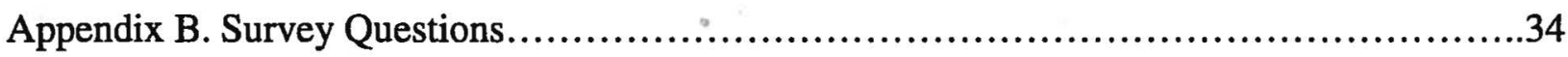


Medication Adherence Factors Among Hypertensive Nigerian Immigrants

Hypertension is a dangerous health condition and has a high prevalence among the general population (Center for Disease Control and Prevention [CDC], 2014). It is generally defined as systolic blood pressure higher than $140 \mathrm{~mm} \mathrm{Hg}$ and a diastolic greater than $90 \mathrm{~mm} \mathrm{Hg}$ (Merriam-Webster, 2015). It is also the most prevalent cause of stroke and kidney failure (American Society of Hypertension, 2014), and contributes to heart disease and stroke, which are two leading causes of death (CDC, 2014). Hypertension is considered a primary or contributing cause of $15 \%$ of deaths in the United States (U.S.) (Go et al., 2014). The prevalence of hypertension in the U.S. is estimated to be about $33 \%$ of the population (CDC, 2014). The American Heart Association (AHA, 2014) estimates that 80 million Americans have been diagnosed with hypertension. There are known disparities in the prevalence among different racial and ethnic groups, with non-Hispanic blacks ranking highest in this disease (CDC, 2014). The prevalence increases to $40 \%$ within the African American population (CDC, 2014). According to the AHA, every African American has a high chance of becoming, or has a relative that is, hypertensive. In the Global Status Report on noncommunicable diseases by the World Health Organization (WHO, 2011), the African region has the highest incidence of hypertension in the world at the rate of $46 \%$. On the new Global Status Report on hypertension in Africa by Vijver et al. (2013), it was noted that cardiovascular disease has taken over as the number one cause of death in Africa and is expected to increase in the next decade.

Pharmacological therapy is initiated in the management of hypertension when lifestyle modification is inadequate to control high blood pressure and over $50 \%$ of these patients will require more than one type of medication (Madhur, 2014). According to Gu, Burt, Dillon, and Yoon (2012), the use of antihypertensive medication increased significantly in the last decade 
and uncontrolled hypertension rates persist among older Americans and non-Hispanic blacks. Many individuals require two or more medications to reduce and maintain their blood pressure (Gu et al., 2012), which may be a contributory factor to the level of nonadherence to hypertensive medication therapy. The CDC (2013) defines medication adherence as a patient conforming to recommendations from the provider on timing, dosage and frequency during the time prescribed. About 50\% of hypertensive Americans do not have their blood pressure under control, up to $30 \%$ of prescriptions are not even filled, and $50 \%$ of medications that are filled are not continued as prescribed (CDC, 2013).

The cost of hypertension treatment in 2008 was estimated to be about $\$ 69$ billion (Go et al., 2014). The mean expenditure per person for the treatment of hypertension was higher for Hispanics and non-Hispanic blacks ( $\$ 981$ and $\$ 887$, respectively), than for non-Hispanic whites (\$679) (Go, et al, 2014). Kaplan, (2015) cited the lack of patient adherence with hypertension management as the main reason for poor blood pressure control. Whilst education, quality improvement efforts, and resources for the management of hypertension have been available for decades, medication adherence continues to be a challenge (Go et al., 2014). The purpose of this qualitative study is to explore the reasons why hypertensive patients, who are Nigerian immigrants, may not adhere to their medication management. The sample population was drawn from Nigerian immigrants in Sacramento, California. The reasons that contributed to nonadherence, such as the influence of culture, religion, and acculturation in management of hypertension, were investigated.

Significance. Hypertension is one of the leading health indicators noted by Healthy People 2020 (HP2020) to have shown improvement (HP2020, 2014), and it is one of the modifiable major risk factors that cause heart disease and stroke. It accounted for $\$ 500$ billion in 
healthcare and related expenditure in 2010 (HP2020, 2014). The Center for Medicare and Medicaid Services (CMS, 2013) has suggested some ways to curb nonadherence: understand the predictors and address them, and ask patients specific questions related to their beliefs and cultural norms about taking medications.

This study explored the reasons why Nigerian immigrants did not adhere to their medication management and the influence culture and religion had in the management of hypertension. Several studies exist that investigated the reasons for nonadherence among populations, including Nigerians, and what influence culture may have on medication management. But no study was found for Nigerian immigrants living in the U.S. Results from this study will contribute to nursing knowledge on culturally sensitive interventions pursuant to an increase in compliance and providing direction for future nursing research.

The research questions posed are the following:

1. How do Nigerian immigrants explain the cause of hypertension and how do culture and religion impact their belief system in the cause and management of hypertension?

2. What are the explanations that influence the adherence to hypertension medication among Nigerian immigrants?

\section{Theoretical Framework}

The Neuman systems model on nursing theory provided the framework for this study. Neuman's model has transformed through the decades to become a flexible framework that examines the relationship between nursing interventions and how patients respond to stressors in their environment (Whetsell, Gonzalez, \& Moreno-Fergusson, 2015). As a grand theory, Neuman systems model is comprehensive, and can be generalized to diverse settings, human experiences, and across cultures (Whetsell, et al., 2015). Neuman believes in the uniqueness of 
each individual or group. Environmental stressors differ and so does the way they affect individuals and communities (Neuman, 2002). The model assumes that every unique person has an established line of defense against environmental stressors. The interrelationship of physiological, psychological, sociocultural, developmental, and spiritual variables make up the dynamic composition of an individual's wellness or illness state (Neuman, 2002).

Neuman (1995) defined the environment as any internal or external factor that surround the client and may influence the client in a positive or negative way. Environmental stressors are believed to cause instability to the client system with a potential for negative or positive outcome (Neuman, 2002). According to Neuman (1995), the role of the nurse is to provide interventions that are necessary to maintain a stable client system. The nurse is expected to accurately assess the environmental stressors and assist the client to make adjustments to move toward a healthy state.

Neuman systems model has been used as a theoretical framework in several studies. Talato (2014) used Neuman systems model as a theoretical framework in a study on the prevalence and risk factors for prehypertension among adults in Burkina Faso. The five variables in the Neuman systems model were found to validate the relationship of the variables on

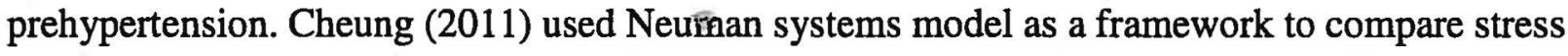
levels among homeless people in three different homeless shelters and to identify methods of nursing intervention. The three levels of nursing intervention were found to be applicable to the management of prehypertension in homeless people.

Application of Neuman's model. Neuman's model is a flexible framework that examines the relationship between nursing interventions and how patients responded to stressors in their environment (Whetsell, et al., 2015). The Nigerian immigrant (client) is considered an 
open system that interacts with, and reacts to, the stressors in its environment. Each person is bound by certain genetic relationships, cultural preferences, and ways they respond to stress. Environmental stressors are believed to cause instability to the client system, with a potential for negative or positive outcome. Living with high blood pressure is a stressor and may break the normal and flexible line of defense for Nigerian immigrants. The Nigerian immigrant has unique cultural and religious beliefs that may or may not contribute to their ability to create resistance against stressors. The effect of the environment on individuals will be considered as a contributor to stress and hypertension. This study will aim to discover what unique cultural compositions the select population has that may be impeding protection from environmental stressors.

\section{Literature Review}

The purpose of this study and many capstone projects are to improve healthcare outcomes for specific populations. We are in a generation that is increasing the use of evidence in the provision of care. Existing evidence in literature was reviewed in order to provide a balanced view of hypertension and medication adherence. Abel (2011) conducted a crosssectional, correlational nonexperimental study on issues that influence medication adherence in black women with hypertension. The research question focused on the differences in the background variables of Black women who adhered to medication management and those who did not. The author chose a sample of 80 Black women between 18 and 80 years old, who were taking antihypertensive prescription medications for blood pressure control. Findings from the study did not show a marked difference between those who adhered to antihypertensive medications and those who did not. Age of participants and trust in healthcare provider were found to be the only variables that predicted adherence to medication. 
Ogedegbe, Harrison, Robbins, Mancuso, and Allegrante (2004) conducted a qualitative study to explore the perspectives of hypertensive African Americans regarding perceived barriers to prescribed antihypertensive medication adherence. The interview questions focused on the challenges participants faced in adhering to their medication along with the things that made it easy or difficult for them to remember. Use of reminders, having a routine, knowledge about hypertension treatment and complications, and having social support were found to facilitate medication adherence. The barriers were grouped as patient-specific, medication-specific, logistic, and disease-specific barriers. Another study was conducted by Segbefia, OwareGyekye, and Akpalu (2012), to explore the views and beliefs of hypertensive patients in Ghana regarding the management of their condition. The qualitative study used an exploratory and descriptive design. A purposive sampling method was used to select 12 participants who ranged from 45 to 60 years of age with confirmed diagnosis of hypertension for at least one year. Findings from the study revealed that participants' knowledge about their hypertension was tied to supernatural beliefs and the reasons for nonadherence were mainly forgetfulness and their outlook in life. Boima et al. (2015) conducted a study to determine factors associated with medication nonadherence among hypertensive patients in Ghana and Nigeria. A cross-sectional study was done with 357 hypertensive patients. Findings showed that nonadherence was high (67\%) and was also associated with depression.

\section{Rationale for the Study}

The necessity of this capstone project was to provide information related to a vulnerable population that is yet unstudied. The primary studies reviewed used both qualitative and quantitative approaches and looked at hypertension and medication adherence in the African and African American population. Nigerian immigrants experience acculturation after they arrive in 
the U.S.; where they have better opportunities for advanced healthcare and availability of medications than those living in Africa. There were no studies found that had evaluated the perceptions of Nigerian immigrants in medication adherence. The sample population used in this study has better opportunities for advanced healthcare than their cultural counterparts in Nigeria and Africa, including availability of antihypertensive medications and a higher level of education. They receive healthcare from providers that want to provide patient-centered care but may lack understanding of the influence their culture, belief systems, and knowledge of the disease process may have on their ability to conform to prescribed medication management. It therefore becomes necessary to study this population.

\section{Method}

A descriptive phenomenological approach was used for this research project with the goal to understand perceptions about medication adherence and the influence of culture and religion on hypertension management. Studies with a phenomenological approach focus on the experiences that vary from thought, perception, imagination, desire, memory, social activity, and personal awareness (Smith, 2013). Interview questions were bracketed and asked in an openended manner. Ideas and use of language that suggested preconception of the experience were avoided. The need for audio recording of the conversation was explained in the informed consent document.

\section{Participants}

The required participants were selected by use of a purposive sampling method. The sample population was drawn from Nigerian immigrants living in Sacramento, California, with a healthcare confirmed diagnosis of hypertension, and who were taking antihypertensive medications. Nigerian immigrants are described as persons who were born in Nigeria and 
immigrated to the U.S. after the age of 18 . All participants verbally confirmed their medical diagnosis of hypertension and the investigator visually inspected their most recently filled prescription bottle. All subjects were anonymous as the interviews were done individually and the data document was coded to protect their identities. Melynk and Fineout-Overholt (2011) suggest that deciding the optimum sample size should be based on number of interviews needed before the concept of redundancy or saturation is achieved. A total of nine participants were sampled and interviewed for the study (six males, three females, Mage $=53$ years, age range: 42 71 years). Five participants were between the ages of 42 and 49, two were between 50 and 59, and two participants were above 60 years of age. Two of the participants had associate degrees, seven completed university education, and three had postgraduate degrees. One participant was retired and the rest were employed full-time. Four participants were civil servants with the State of California, two participants worked for the health care industry, and two were employed with private entities. All nine participants identified themselves as Christians.

\section{Procedure}

Ethical considerations. Ethical clearance was obtained from California State University, Fresno, along with informed consent from the participants. The need for audio recording of the conversation was explained in the informed consent document and also verbally to the participants.

Data collection. Semi-structured individual interviews were conducted to address exploratory models of hypertension medication adherence. The questions focused on the participants' understanding of the cause of their hypertension, cultural beliefs, perceptions, and the influence of prayer on illness and adherence. Other questions included factors that influenced adherence/nonadherence, financial burden of medication, and side effects/complications of medication. Information was also obtained on the psychology and fears related to having 
hypertension; along with the effect of living in the U.S. has on disease management. The questions were developed after careful review of the literature. Data was collected from August 1,2015 , through October 30,2015 . The interviews were scheduled and conducted by the principle investigator. Each participant was interviewed once during the data collection period and the interview time lasted about 25-35 minutes. A $\$ 15$ gift card to a well-known healthy juice store was provided to each participant in appreciation of his or her time. Responses were transcribed and a thematic analysis was performed.

Data analysis. Colaizzi's seven-step approach to qualitative data analysis was used in this study (Edward \& Welch, 2011). Colaizzi's phenomenological method requires the transcription of audio recorded data, extraction of significant statements, and formulation of meanings that emerge based on insight. Furthermore, data is clustered into themes, results integrated into a description of the topic, and an essential structure of the phenomenon is formulated. The final step of the Colaizzi approach is to return to the participants to confirm the descriptive results and analysis of their experiences (Edward \& Welch, 2011). All participant interviews were recorded and transcribed. Each participant's description of his or her feelings was read to provide a general sense of understanding and extraction of statements that lend themselves to the research questions and then the results were compiled. Direct quotations from participants were included for an accurate reflection of data and of the meaning of the statements subjects articulated in order to create themes and categories. In following the Heideggerian phenomenological approach, the principle investigator's cultural understanding of the experiences of the participants guided the bracketing of the themes and aided in interpreting the experiences of the participants. Reflection and analysis of the data sought similar themes and concepts in the interviews. The final steps of Colaizzi's approach suggest the researcher returns 
to each participant for validation of the results. Participants were contacted and validated the included statements. To further ensure validity and rigor, a project committee member, who is familiar with the culture and shares the same ethnic background, read the transcript for consensual validation. The project chair who supervised the study is experienced in qualitative methodology and was consulted throughout the process.

\section{Results}

The findings of the study are grouped into categories as described below:

\section{Length of Time From Diagnosis}

Two participants reported they were diagnosed with hypertension in the past year, three were diagnosed within the past 10 years and another three have been hypertensive for over 20 years. Participant \#1 reported, "I will say that I have had hypertension for 20 years and has taken medications for 20 years as well." Two of the participants discovered their hypertension during mandated health screening before visa approval to come to the U.S. Participant \# 2 reported how he found out about his high blood pressure, "When I went to the embassy in Nigeria as I was preparing to come to the United States. That was the first time I knew my BP was high. From that moment on, they recommended that I take blood pressure medications. It appears that once you get on that journey of taking medicines, there is no going back." Another participant discovered his diagnosis during a work-mandated physical examination, and two participants had a near catastrophic event that led to their diagnosis.

\section{Causes of Hypertension}

Participant responses varied with regard to their belief on the causes of hypertension. Most of the participants attributed the cause to genetics and others considered stress and diet to be the causes. Participant \#6 reported, "Mine is hereditary. Both of my parents and all my 
siblings has high blood pressure." Participant \# 8 noted, "Well, from what I understand, I think it is genetic. My mother had high BP; I don't think my siblings has it but each one is taking one form of medication or the other." Participant \# 2 reported he researched his family history and can conclusively attribute the cause of his hypertension to genetics. According to his recollection "My hypertension is inherited and I will tell you why and you will find it interesting. My grandmother died in her 40's and no one knew why. She was kind of a plump woman. Nobody knew then what we know now that she probably had hypertension. My uncle died of renal disease that it was attributed to hypertension. My father and his siblings without exception had hypertension and now my son is also hypertensive. I know he got it from me. I narrowed the source down to my paternal grandmother. That's where it all came from." Two other participants attributed the cause to their type of diet, lack of consistent exercise, and stress. Participant \#9 reported, "Food was something I did not pay attention to. I took care of family and fed my husband well, but I fed myself with leftovers." Another participant reported, "I will say my hypertension is caused by the type of diet I eat; I will not attribute mine to stress."

\section{Warning Signs of Elevated Blood Pressure}

The participants were asked what they considered to be warning signs that their blood pressure was elevated. Many indicated headaches, loss of appetite, and heart palpitations. Participant \# 6 reported, "I have a weird kind of headache and sometimes if I don't have adequate sleep, my blood pressure goes up." Participant \# 9 reported, "Getting angry and being upset aggravates it for me. I experience what seems as a blood rush into my head, my heart beats faster. I have palpitation and sudden dizziness and have experienced that twice and when I checked, my blood pressure, it was high." Almost all the participants reported mild or severe 
headaches as their warning sign, and related diet control, relaxation, and taking their medication as ways to control their blood pressure.

\section{Medication Adherence}

The participants were asked if they took their medication as prescribed and what influenced their adherence. Most participants reported not taking their medication exactly as prescribed by their physician and cited several reasons why they were not adherent. Participant \#6 reports, "There are so many times that I don't take my medication as it was prescribed. If I go out of town, I may forget to take my medication or sometimes, I just don't feel like taking it." Participant \#5 reported, "I was not taking it as prescribed previously and was doing diet and exercise, with the hope that it will come down. It did not; and now I take it regularly." One of the participants who reported adherence attributed it to a prior catastrophic experience. "I have not missed my medication since the year I had my medical crisis. I take it like I read my bible. It is in my pocket wherever I go, whether I am travelling or going to a party. Considering what I went through, I do not want to repeat the experience." Participant \# 6 reported that he does not take his medication when he is going through a religious fast.

\section{Cultural Influence}

Some participants cited strong cultural or religious beliefs and others refuted any influence. Many participants attributed their education to be the reason why they no longer held cultural beliefs to the cause of their illness. Participant \# 2 reported, "When my grandmother had a stroke, nobody knew what it was at the time. They thought that it was what we call 'mba mmuo,' which meant that the 'spirits' took hold of her or somebody placed a voodoo in her farm. Back home, no one dies naturally. Something or someone is always responsible. My doctors have explained the cause of hypertension to me. Luckily for me also, I am educated and I know 
better. The moment I ruled out that no one had any hand in my ailment, it enabled me to focus on what I needed to do." Another participant shared, "People think it is caused by voodoo and that somebody gave them high blood pressure; especially where I come from (Nigeria) but I don't believe in that." Another participant referred to other people believing that "their illness is caused by somebody or unforeseen forces."

\section{Influence of Religion}

All the participants reported that they were Christians and believed in prayer. They were asked what influence prayer had on the management of their hypertension. Participant \#9 reported, "Religion can be a double-edged sword and it depends on how it is utilized. God has given us wisdom and he has given medical practitioners wisdom to heal. I cannot operate on a blind faith. People die prematurely because when they have a condition requiring medical intervention, they resort to mere prayer. Prayer and meditation in the word of God help me heal emotionally. In addition to my religion, I do what need to be done to get better. A lot of our people has taken religiosity to an extreme." Others also believed that prayer alone cannot heal their hypertension. Many acknowledged the use of prayer, but also stressed the importance of taking their medication. Participant \#2 reported, "Prayer has a calming effect. I pray and take my medication as well. I do not wear my religion on my sleeve." Two participants acknowledged they did not hold any religious beliefs with regard to their illness.

\section{Effects of Medication on Sexual Life}

The participants were asked if the hypertensive medication affected their sexual life and if that impacted their adherence to their medications. All the female participants denied any significant side effects and reported no negative impact of hypertensive medication on their sexual life. Participant \#6 reported no side effects and attributed sexual disturbances to only 
males. "I have not had that experience. I read about it and it affects men and not women." Most of the male participants reported sexual side effects with their medications and some attributed it to the reason for medication nonadherence. Participant \#8 reported he stopped taking his medication because it was affecting his sexual life. His spouse encouraged him to restart taking it and seek alternative medication from the physician. Participant \#3 reported when he complained to his physician, he was told that other men have the same experience, and as long as he was still able to "perform," he should manage. Participant \# 2 reported erectile dysfunction and impotence with one of his medications and thus was later placed on another medication. He reported that he researches the side effects of his medications on the Internet before taking any new prescription. "I go on the Internet and research it and if they say it's going to cause a problem in the bed, I throw it away and I will go to the doctor and ask for something better." One participant reported higher sexual performance since being on his antihypertensive.

\section{Fears About Hypertension}

The participants were asked if the fear of associated complications or dying were the motivation to maintain adherence to medication management. Many reported the fear of debilitating illness and provided stroke and renal disease as examples. Participant \# 5 reported the fear of "stroke and heart attack make me take my medication as it is prescribed." Participant \#1 reported he is afraid of renal failure and does not want to go on dialysis.

\section{Being in the United States}

The participants were asked how their acculturation in the U.S. has affected their hypertension diagnosis. Most participants considered it as an advantage, especially with medication availability and affordability. Participant \# 1 reported, "Being in the United States obviously has a lot of advantages because the medications are available with the healthcare 
coverage that I have." Participant \# 5 noted residing in the U.S. is a positive thing, as she does not have to worry about receiving fake medication. Many participants also reported the negative impact of living in the U.S. on their disease. Many participants however reported a higher level of stress with living in the U.S. Participant \# 5 reported, "The stress level here in the U.S. has contributed to me being hypertensive." Another participant reported "There is too much running around and being in debt in so many things. Living here results in lack of regular communal associations/visits with family and friends. There is more isolated living than the way we lived at home."

\section{Discussion}

The focus of this capstone project was to explore the perceptions of hypertensive Nigerian immigrants to the cause of their illness and the factors that contributed to nonadherence such as the influence of culture, religion, and acculturation in the management of hypertension. The findings will be discussed based on the research questions that were posed previously.

\section{How do Nigerian immigrants explain the cause of hypertension and how does culture and religion impact their belief system in the cause and management of hypertension?}

Findings from this study showed that participants in the study credited the cause of their hypertension to genetics, stress, and diet. Taylor et al. (2012), reported similar perceptions among Nigerian indigenes. In their study of explanatory models of hypertension among inpatients in a Nigerian hospital, their participants used phrases such as "thinking too much" as a cause of hypertension in Nigeria. Segbefia et al. (2012) reported similar belief systems, stress, heredity, and poor diet, as the main causes of hypertension in another study of Ghanaian citizens (another African country). The understanding of the causes of hypertension by Nigerian immigrants is similar to those held by Nigerians living in their native land and other African 
countries as evidenced by Taylor et al. (2012). The beliefs about hypertension cause are also similar to the risk factors associated with hypertension by the AHA (2015). Family history, poor diet, and stress are among the factors that contribute to hypertension.

While many participants acknowledged the existence of cultural beliefs to causes and management of hypertension, western education was strongly cited as the reason for dispelling such beliefs. Many stated their friends or other people they knew still held strong cultural beliefs. Prior to western education, people traditionally believed that someone either caused their illness or evil, unseen forces were involved. Religion was attributed to playing a major role in psychological health, yet many reported prayer was not sufficient to heal hypertension. They acknowledged the practice of prayer, but also stressed the importance of taking their medication. The reason for this change in belief system was also attributed to education. Participants made references to others as holding beliefs that God can heal their hypertension. Osamor and Owumi (2010) reported the use of complementary and alternative medicine in the management of hypertension among those who believe in the supernatural causes of hypertension in urban Nigeria. Just like hypertension, other studies revealed the belief system of supernatural causes of other illnesses, such as stroke and mental illness.

Some participants considered living in the U.S. an advantage, especially with medication availability and affordability. Others reported the negative impact of living in the U.S. on their disease. A higher level of stress was noted as a negative impact. The participants easily attributed negative side effects, attitudes, and beliefs to "other" people. Most participants reported that they know "someone" who is not following their medication regimen, is experiencing side effects, and has negative cultural beliefs, but were unwilling to share their story.

Nigerian immigrants reported headaches, loss of appetite, and heart palpitations as 
indications of elevated blood pressure and the reliance on these warning signs before taking action. Taylor et al. (2012) reported similar symptoms in their study conducted in Nigeria. The AHA reports evidence does not support that high blood pressure causes headaches, except when in a hypertension crisis. Headaches therefore cannot be relied on as a valid indicator that blood pressure is elevated. Reliance on a headache as an indicator to elevated blood pressure will predispose Nigerian immigrants to catastrophic events.

\section{What are the explanations that influence the adherence to hypertension medication among Nigerian immigrants?}

This study revealed that most participants are nonadherent to their medication. Several reasons cited were forgetfulness, being away from their home environment, self-management, and medication side effects. Sexual side effects to hypertensive medications among men were found to be a major impediment to adherence. Participants reported erectile dysfunction, loss of sexual drive, and impotence as some side effects they have experienced. Factors associated with nonadherence are multifaceted and the issue of sexual-related side effects cannot be overlooked among Nigerian men. This study revealed that many men will not voluntarily discuss sexual dysfunction and will not maintain their medication regimen as a result. In the Nigerian culture, men have difficulty discussing their sexual concerns. As stated by one of the participants, "We Nigerian men are secretive about telling anyone we cannot perform in bed. I trust you and that's why I am discussing this." Another participant said, "I don't know if it is proper to discuss the fact that I had problems in bed when I started taking my medications." In the Nigerian culture, sexual performance is highly linked to masculinity. Segbefia et al. (2012) reported the same cultural inclination among Ghanaians.

This study found fear of debilitating disease to be a strong motivator for medication 
adherence. Two participants reported they had close friends who had a recent stroke after they stopped taking their hypertensive medication as they "could not perform in bed." Another participant expressed surviving a "catastrophic" event and so, pledged to keep to the medication regimen. Boima et al. (2015) suggested that despite the level of education, all patients need education on hypertension management, side effects of their medication, and what to do when they have side effects.

\section{Linking Theory to Practice}

The use of theory in practice is to assist us to better understand patients' conditions and plan the needed interventions. As Eldridge (2015) described, applying theory in practice improves nursing care by giving it structure, helps to achieve congruence between process and product, and provides us with a framework to review the effectiveness of our interventions. The Neuman systems model on nursing theory was used as the theoretical framework to examine how patients responded to stressors in their environment. It assumes that each unique individual will respond to the stress of the environment based on their line of defense. Environment may influence the client in a positive or negative way. Hypertension was found among the participants to be a stressor that caused disruption in the human equilibrium. Many participants listed genetics and stress as causes of their hypertension. Living in the U.S. contributed to their stress and yet also served as a line of defense. The line of resistance, as described by Neuman, is those protection factors that are activated for use in resisting increases in stress. Many participants reported the use of exercise and prayer for management of their illness.

The model describes internal environment as those factors within the client system (culture), while external environment as the factors outside of the client system. Environmental stressors are believed to cause instability to the client system with a potential for negative or 
positive outcome (Neuman, 2002). The study looked at how the environment as a variable, influenced the line of defense for the Nigerian immigrant. Living in the U.S. and acculturation was referenced as both a positive and negative influence on health. Some participants considered the positive nature of medication availability, while others considered the change in living location as adding to the stress of hypertension.

As previously noted, the Nigerian immigrant has unique sociocultural and religious beliefs that contribute to their ability to create an innate line of resistance against stressors and to assist in recovery from stress factors. In general, the Nigerian community has strong religious beliefs and practices, and this was also found to be true among the study participants. Many reported the use of prayer for psychological healing and cultural beliefs, but did not report it as an influence on medication adherence. Many stated education and living in the U.S. had more bearing on how they managed their hypertension. There was strong cultural influence on how participants discussed either their disease or the side effects from their medication, which in turn affected their adherence. As noted by two participants, "The Nigerian culture does not allow us to talk about our illness freely. We hide what we have." "Our culture play a role in whatever we do whether we like it or not and influence whether we talk about our illness or not." The role of the nurse in Neuman's model is to assist individuals primarily or secondarily to maintain their state of wellbeing. It is therefore important that the nurse understands this cultural variable and plan interventions that will foster a positive nurse-patient relationship and build trust in order to support and encourage honest communication among this select population.

\section{Limitations}

The sample size for this study was nine. This qualitative study had the goal to reach 1015 participants or until saturation was reached. Despite several efforts to attract voluntary 
participants, Nigerian immigrants were reluctant to discuss their personal health issues. The investigator made numerous efforts to recruit more participants without success. The study criteria were limited to secondary education, an age range of 18 to 75 , and use of English language. Older hypertensive patients that were more immersed with the Nigerian culture may have provided more culturally-qualified information. Those who agreed to participate in the study may not accurately reflect the population of the Nigerian immigrant. They also may not have expressed all the facts and issues related to their belief systems and accurate medication adherence, as evidenced by the frequent reference to "someone" they knew. Audio recording was included as part of the data collection and this disclosure was made to the participants. Despite the reasons why the interview needed to be recorded, participants were often open to discuss more when the recorder was turned off, and thus limited the use of information gathered after the interviews. With the small sample size, this study may not have external validity and be generalized to other populations.

\section{Implications for Nursing Practice}

Hypertension prevalence is high among the African community. Unmanaged hypertension leads to devastating and debilitating conditions such as stroke and renal failure. Results from this study can help serve as a guide to nursing practice. Nurses must develop skills to discuss coping strategies with their patients. Participants from this and other studies link hypertension to stress. Every encounter that a nurse has with the immigrant patient is an opportunity to address methods to cope with stress. Apart from the stress of daily living and caring for themselves and their nuclear family, many immigrants are burdened with making money to care for their family members that they left behind in Nigeria. Patients must have stress management strategies to help lower their blood pressure. Stress management should be 
incorporated as part of the treatment regimen for hypertension. Results from the study make it clear that culturally, Nigerian immigrants have difficulty sharing about personal health disabilities. Understanding this attitude makes it imperative for nursing to build a trusting relationship with this population of patients.

Sexual performance is of extreme importance and is linked to masculinity among Nigerian men. Because erectile dysfunction is a side effect of hypertensive medication, it negatively impacts medication adherence among Nigerian men. This is compounded by the cultural difficulty of discussing personal health disabilities with others. As was discovered by this study, many patients may stop taking their medications and experience a catastrophic event due to their limited understanding of available options to manage the issue of erectile dysfunction as a medication side effect. Healthcare providers should do more in explaining medication side effects and assure patients of the availability of options when they do occur. The use of communication techniques in supporting patients to discuss their illnesses, fears, and side effects they are experiencing should be encouraged among health care providers.

Specific information related to sexual side effects should be discussed. Many participants denied having any side effects to their medication, until the question was specifically asked about effects medication has on sexual performance. Healthcare providers may consider modifications to sexual health history with this population and rephrase standard questions to obtain accurate history.

\section{Recommendations for Future Research}

There were no studies found on hypertension management and medication adherence among Nigerian immigrants. Literature on medication adherence and cultural beliefs of 
Nigerians and other African countries will continue to emerge in the future. The incidence of noncommunicable disease is rising within the African continent, and despite education status, medication adherence is low and is attributable to side effects of hypertensive medication. This study confirms the literature of low medication adherence among hypertensive patients and that there are cultural influences to medication adherence. It also supports the fact that adherence will improve with effective communication with healthcare providers. Given the side effect of hypertensive medication on sexual performance and the resultant medication nonadherence in males, further study may provide insight on the effect of spousal support on medication adherence among African male immigrants. The cultural influence on Nigerians immigrants supports minimal disclosure of health and personal information and many also reported a high level of stress associated with living in the United States. Future studies may focus on the presence and disclosure of mental illness to their healthcare providers by Nigerian immigrants with hypertension. 


\section{References}

Abel, W. M. (2011). Issues influencing medication adherence in Black women with hypertension (Doctoral Dissertation, The University of North Carolina at Greensboro). Retrieved from http://libres.uncg.edu/ir/uncg/f/Abel_uncg 0154D_10814(1).pdf

American Heart Association. (2014). Understand your risk for high blood pressure. Retrieved from http://www.heart.org/HEARTORG/Conditions/HighBloodPressure/UnderstandYourRiskf orHighBloodPressure/Understand-Your-Risk-for-High-BloodPressure UCM 002052 Article.jsp

Boima, V., Ademola, A., Odusola, A., Agyekum, F., Nwafor, C., Cole, H., ... Tayo, B. (2015). Factors associated with medication nonadherence among hypertensives in Ghana and Nigeria. International Journal of Hypertension, 2015, 1-8. doi:10.1155/2015/205716

Center for Disease Control and Prevention. (2013). Medication Adherence. Retrieved from http://www.cdc.gov/primarycare/materials/medication/docs/medication-adherence01ccd.pdf

Center for Disease Control and Prevention. (2014). High blood pressure. Retrieved from http://www.cdc.gov/bloodpressure/

Center for Medicaid \& Medicare. (2013). 2014 Clinical quality measures (CQMs) adult recommended core measures. Retrieved from http://www.cms.gov/Regulations-andGuidance/Legislation/EHRIncentivePrograms/Downloads/2014 CQM AdultRecommen d CoreSetTable.pdf 
Cheung, H. (2011). Study of stress, housing program use, and nursing usage among homeless in Chicago (Doctoral dissertation, Loyola University, Chicago). Retrieved from http://ecommons.luc.edu/luc diss/200/

Edward, K. \& Welch, T. (2011). The extension of Colaizzi's method of phenomenological enquiry. Contemporary Nurse: a Journal for the Australian Nursing Profession, October 1, 2011. Retrieved from http://www.readperiodicals.com/201110/2701126981.html\#ixzz3UVDYloxu

Editorial: "Translating Hypertension Research into Effective Treatment for Patients" [Editorial]. (2014). American Society of Hypertension. Retrieved from http://www.ash-us.org Eldridge, C. (2015) Nursing Science and Theory: Scientific Underpinnings for Practice. In M.E. Zaccagnini \& K.W. White (Eds.), The doctor of nursing practice essentials: A new model for advanced practice nursing ( $2^{\text {nd }}$ ed., pp. 3-36). Burlington, MA: Jones \& Bartlett Learning.

Go, A.S., Bauman, M., King, S.M., Fonarow, G.C., Lawrence, W., William, K.A., \& Sanchez, E. (2014). An effective approach to high blood pressure control: a science advisory from the American Heart Association, the American College of Cardiology, and the Centers for Disease Control and Prevention. Hypertension. 2014(63), 878-885. doi: 10.1161/HYP.0000000000000003

Gu, Q., Burt V., Dillon, C., \& Yoon, S. (2012). Trends in antihypertensive medication use and blood pressure control among United States adults with hypertension: The National Health and Nutrition Examination Survey, 2001 to 2010. Circulation. 2012(126), 21052114. doi: 10.1161/CIRCULATIONAHA.112.096.156

Healthy People 2020 (2014). Heart disease and stroke. Retrieved from 
https://www.healthypeople.gov/2020/topics-objectives/topic/heart-disease-andstroke/objectives

Healthy People 2020 (2014). Leading health indicators: Progress update executive summary. Retrieved from https://www.healthypeople.gov/sites/default/files/LHI-ProgressReportExecSum 0.pdf

Hypertension. (n.d.). In Merriam-Webster's online dictionary (2015 ed.). Retrieved from http://www.merriam-webster.com/dictionary/hypertension

Kaplan, N. (2015). Patient adherence and the treatment of hypertension. UpToDate. Retrieved from http://www.uptodate.com/contents/patient-adherence-and-the-treatment-ofhypertension

Madhur, M.S. (2014). Hypertension treatment \& management. Medscape. Retrieved from http://emedicine.medscape.com/article/241381-treatment

Melnyk, B.M., \& Fineout-Overholt, E. (2011). Evidenced-based practice in nursing and healthcare: A guide to practice. Philadelphia, PA: Wolters Kluwer/Lippincott Williams \& Wilkins.

Neuman, B. (1995). Systems and Nursing: Conceptualization of the Neuman System Model. In B. Neuman (Ed.), The Neuman Systems Model (3 ${ }^{\text {rd }}$ ed., pp. 3-44). East Norwalk, CT: Appleton \& Lange.

Neuman, B. (2002). The Neuman Systems Model. In B. Neuman \& J. Fawcett (Eds.), The Neuman Systems Model (4 ${ }^{\text {th }}$ ed., pp 3-33). Upper Saddle River, NJ: Prentice Hall. Ogedegbe. G., Harrison, M., Robbins, L., Mancuso, C. \& Allegrante J. (2004). Barriers and facilitators of medication adherence in hypertensive African Americans: a qualitative study. Ethn Dis 2004; 14: 3-12. 
Osamor, P., \& Owumi, B. (2010). Complementary and alternative medicine in the management of hypertension in an urban Nigerian community. BMC Complementary \& Alternative Medicine, 2010, 1-9. doi:10.1186/1472-6882-10-36

Segbefia, T., Oware-Gyekye, F., \& Akpalu, A. (2012). Management of hypertension: The views of patients at Korle-Bu Teaching Hospital, Accra Ghana. West African Journal Of Nursing, 23(2), 74-83.

Smith, D.W. (2013). Phenomenology. In E.N. Zalta (Ed.), The Stanford encyclopedia of philosophy (Winter 2013 ed.). Retrieved from http://plato.stanford.edu/entries/phenomenology/

Talato, K. (2014). Prevalence and risk factors for pre-hypertension among adults in Burkina Faso (Doctoral Dissertation, Azusa Pacific University, Azusa). Retrieved from http://gradworks.umi.com/36/14/3614164.html

Taylor, K. D., Adedokun, A., Awobusuyi, O., Adeniran, P., Onyia, E., \& Ogedegbe, G. (2012). Explanatory models of hypertension among Nigerian patients at a University Teaching Hospital. Ethnicity \& Health, 17(6), 615-629 15p. doi:10.1080/13557858.2013.771151

Vijver, S., Akinyi, H., Oti, S., Olajide, A., Agyemang, S., Aboderin, I., \& Kyobutungi, C. (2013) Status report on hypertension in Africa - Consultative review for the 6th Session of the African Union Conference of Ministers of Health on NCD's. The Pan African Medical Journal. 16, 38. doi:10.11604/pamj.2013.16.38.3100

Whetsell, M., Gonzalez, Y., \& Moreno-Fergusson, M. (2015). Economic theories. In J.B. Butts \& K.L. Rich (Eds.), Philosophies and theories for advanced nursing practice ( $2^{\text {nd }}$ ed., pp. 319-338). Burlington, MA: Jones \& Bartlett Learning. 
World Health Organization. (2011). Global status report on non-communicable diseases 2010.

Retrieved from http://www.who.int/nmh/publications/ncd report full en.pdf 


\section{Appendix A}

\section{Informed Consent}

Project Title: Nigerian Immigrants' Perceptions about hypertension medication adherence: A qualitative approach.

Purpose of the Study: You are invited to participate in a study conducted by Dr. Tamara McKinnon, DNP, Assistant Professor at the Valley Foundation School of Nursing, San Jose State University, and Beulah Iroegbu, a doctoral student, licensed by the Board of Registered Nursing in the state of California. We hope to learn the perceptions of Nigerian immigrants toward medication adherence in hypertension management. You were selected as a possible participant in this study because you have identified yourself as having a history of high blood pressure and taking blood pressure medication.

Procedures: If you decide to participate, Dr. McKinnon or Beulah Iroegbu will interview you at a convenient location about your experiences with taking your hypertensive medications. The conversation may last for an hour. The conversation will be recorded on a small tape recorder to make sure that we get all of the details. We may contact you again for a follow-up interview if necessary. You will be asked about 12-16 open-ended questions regarding your lived experience, beliefs and cultural influences regarding your hypertension and how you take your medications.

Potential risks and discomforts: Risks for the study will be minimal. You may experience mild discomfort or anxiety while answering the questions. If there are any questions you do not want to discuss we will omit them. We will stop the interview at any time if you become tired or uncomfortable

Potential benefits: You will have the opportunity to discuss how you feel about taking high blood pressure medication with someone who is interested in this subject. Your participation in this study will contribute to knowledge and understanding of perceptions of Nigerian immigrants who have high blood pressure in medication management. We cannot guarantee, however that you will receive any benefits from this study; but the findings from the study will support nursing and medical knowledge and may also help to develop culturally sensitive interventions, and tailor medication education to fit the needs of Nigerian immigrants. You will receive educational materials and provided educational support following the interview.

Confidentiality: The information you provide will be kept confidential. You will not be personally identified in the study report. The information obtained during this study will be typed, coded and reviewed, and will be kept confidential. Only the primary investigator will have access to your information. All documents will be kept in a locked file box. The results of this research may be published, but your name and medical information will not be used. Your name will not be attached to any typed transcripts. Upon request and at the completion of the study, a written summary of the completed study results will be made available to you. 
Opportunity to Ask Questions: Before agreeing to participate, you may ask questions concerning this study. You may call Beulah Iroegbu at 916-599-4526. Questions regarding the rights of research subjects may be directed to Dr. Constance Jones, Chair, CSUF Committee on the Protection of Human Subjects, (559) 278-4468.

Freedom to Withdraw: If you decide to participate, you are free to withdraw your consent and to discontinue participation at any time without penalty. The Committee on the Protection of Human Subjects at California State University, Fresno has reviewed and approved this research.

You will be given a copy of this form to keep.

YOU ARE MAKING A DECISION WHETHER OR NOT TO PARTICIPATE. YOUR SIGNATURE INDICATES THAT YOU HAVE DECIDED TO PARTICIPATE, HAVING READ THE INFORMATION PROVIDED ABOVE.

With my signature, I grant authorization (permission) for the use and disclosure of my personal information for the purposes of this research study.

Participant Date

Signature of Research

Administering Informed Consent Date

Signature of Person

Investigator/Researcher Date

Signature of Principal

Initials: 
Running Head: HYPERTENSION MEDICATION ADHERENCE

Appendix B

Survey Questions

I.D. number

Date:

1. Tell me about your age, education level and the type of work that you are currently doing?

2. How long have you had high blood pressure and when did your doctor place you on medication?

3. Tell me about your healthcare coverage and availability of your medications to you?

4. Tell me what you believe to be the cause of your illness?

5. When your blood pressure goes up, what signs do you have and what do you do to bring it down

6. Tell me the medications you take and if you take them according to the way it was prescribed all the time?

7. Are there times that you do not take the medication as the doctor prescribed? If yes, what are the reasons, if not what has helped you to be taking them all the time?

8. Explain to me the cultural beliefs you have related to your illness and to your medication?

9. What religious beliefs do you have in relation to your illness and taking your medication?

10. What is the impact that prayer has when you take your medication?

11. When you receive your medication, describe the information you received to the actions and side effects of your medication

12. What is the most side effect of taking your medication and how do you manage that?

13. How does your medication affect your sexual life and how does that impact the way you take your medications?

14. What are your fears about not taking your medications? How does that impact your adherence?

15. How does being in the United States help or not help in the way you take your medication?

16. What other information will you like to share regarding your hypertension and the way you take your medication? 